Ozioma C. Okonkwo,

$\mathrm{PhD}$

Stephanie A. Schultz, BSc Jennifer M. Oh, BSc Jordan Larson, BA Dorothy Edwards, PhD

Dane Cook, PhD

Rebecca Koscik, PhD

Catherine L. Gallagher, MD

N.M. Dowling, PhD

Cynthia M. Carlsson, MD, MS

Barbara B. Bendlin, PhD Asenath LaRue, PhD Howard A. Rowley, MD Brad T. Christian, PhD Sanjay Asthana, MD Bruce P. Hermann, PhD Sterling C. Johnson, PhD Mark A. Sager, MD

Correspondence to Dr. Okonkwo: ozioma@medicine.wisc.edu

\title{
Physical activity attenuates age-related biomarker alterations in preclinical AD
}

\section{ABSTRACT}

Objective: To examine whether engagement in physical activity might favorably alter the agedependent evolution of Alzheimer disease (AD)-related brain and cognitive changes in a cohort of at-risk, late-middle-aged adults.

Methods: Three hundred seventeen enrollees in the Wisconsin Registry for Alzheimer's Prevention underwent T1 MRI; a subset also underwent ${ }^{11} \mathrm{C}$-Pittsburgh compound B-PET ( $\mathrm{n}=186$ ) and ${ }_{18} \mathrm{~F}$-fluorodeoxyglucose-PET $(\mathrm{n}=152)$ imaging. Participants' responses on a self-report measure of current physical activity were used to classify them as either physically active or physically inactive based on American Heart Association guidelines. They also completed a comprehensive neuropsychological battery. Covariate-adjusted regression analyses were used to test whether the adverse effect of age on imaging and cognitive biomarkers was modified by physical activity.

Results: There were significant age $\times$ physical activity interactions for $\beta$-amyloid burden $(p=$ $0.014)$, glucose metabolism ( $p=0.015)$, and hippocampal volume ( $p=0.025$ ) such that, with advancing age, physically active individuals exhibited a lesser degree of biomarker alterations compared with the physically inactive. Similar age $\times$ physical activity interactions were also observed on cognitive domains of Immediate Memory ( $p=0.042)$ and Visuospatial Ability ( $p=$ 0.016). In addition, the physically active group had higher scores on Speed and Flexibility ( $p=$ 0.002) compared with the inactive group.

Conclusions: In a middle-aged, at-risk cohort, a physically active lifestyle is associated with an attenuation of the deleterious influence of age on key biomarkers of AD pathophysiology. However, because our observational, cross-sectional design cannot establish causality, randomized controlled trials/longitudinal studies will be necessary for determining whether midlife participation in structured physical exercise forestalls the development of AD and related disorders in later life. Neurology ${ }^{\circledR}$ 2014;83:1753-1760

\section{GLOSSARY}

$\mathbf{A} \boldsymbol{\beta}=\beta$-amyloid $\mathbf{A} \boldsymbol{\beta} \mathbf{i}=\beta$-amyloid indeterminate; $\mathbf{A D}=$ Alzheimer disease; $\mathbf{D V R}=$ distribution volume ratio; $\mathbf{F D G}={ }^{18} \mathrm{~F}-$ fluorodeoxyglucose; $\mathbf{F H}$ = parental family history; $\mathbf{M E T}=$ metabolic equivalent; $\mathbf{M N I}=$ Montreal Neurological Institute; PCC $=$ posterior cingulate cortex; PiB $={ }^{11} \mathrm{C}$-Pittsburgh compound B; WRAP = Wisconsin Registry for Alzheimer's Prevention.

Animal models of Alzheimer disease $(\mathrm{AD})$ show that physical exercise represents an efficacious means for favorably altering not only cognitive trajectories but also underlying pathophysiologic processes, including $\beta$-amyloid $(\mathrm{A} \beta)$ burden, tau phosphorylation, and neuronal loss. ${ }^{1-3}$ Whereas the beneficial effects of exercise on cognition in older adult humans have been well studied, ${ }^{4}$ investigations of the influence of exercise on AD biomarkers have only just begun. The emerging evidence suggests that higher levels of physical exercise/fitness are associated with preserved brain volume in $\mathrm{AD}$-vulnerable structures, ${ }^{5-7}$ reduced $\mathrm{A} \beta$ burden, ${ }^{8-10}$ and possibly lowered intracellular tau protein ${ }^{8}$ (but see references 11 and 12 for divergent findings). Of note, an evolving body of work indicates that exercise modifies age-associated alterations in

From the Geriatric Research Education and Clinical Center (O.C.O., S.A.S., J.M.O., J.L., D.C., C.L.G., C.M.C., B.B.B., S.A., S.C.J.), William S. Middleton Memorial VA Hospital, Madison WI; Wisconsin Alzheimer's Institute (O.C.O., D.E., R.K., B.B.B., A.L., S.A., B.P.H., S.C.J., M.A.S.), Wisconsin Alzheimer's Disease Research Center (O.C.O., S.A.S., J.M.O., J.L., D.E., C.L.G., N.M.D., C.M.C., B.B.B., H.A.R., B.T.C., S.A., B.P.H., S.C.J., M.A.S.), Departments of Kinesiology (D.E., D.C.), Neurology (C.L.G.), Biostatistics \& Medical Informatics (N.M.D., B.P.H.), Radiology (H.A.R.), and Medical Physics (B.T.C.), University of Wisconsin School of Medicine and Public Health, Madison.

Go to Neurology.org for full disclosures. Funding information and disclosures deemed relevant by the authors, if any, are provided at the end of the article. 
AD-vulnerable brain structures. ${ }^{2,13,14}$ This is of considerable significance given that age is widely recognized as the most critical determinant of the accumulation of $\mathrm{AD}$-related pathology, ${ }^{15,16}$ and the strongest risk factor for the ultimate manifestation of symptomatic AD. ${ }^{17,18}$ If indeed physical activity ameliorates age-related changes in $\mathrm{AD}$ biomarkers, it raises the exciting possibility that exercise might have a pivotal role in the prevention of $\mathrm{AD}$.

In this study, we investigated whether engagement in physical activity attenuates age-associated alterations in fibrillar $A \beta$, cerebral glucose metabolism, hippocampal volume, and cognitive function - core biomarkers of the $\mathrm{AD}$ pathophysiologic process $^{19}$ - in a cohort of at-risk, late-middle-aged adults. We begin by first comparing the relative influence of age, parental family history $(\mathrm{FH})$ of $\mathrm{AD}$, and $A P O E$ $\varepsilon 4$ - the 3 established risk factors for $\mathrm{AD}$-on interindividual variation in these biomarkers.

METHODS Participants. Three hundred seventeen cognitively normal adults from the Wisconsin Registry for Alzheimer's Prevention (WRAP) participated in this study. The WRAP is a longitudinal registry of approximately 1,500 middle-aged adults who were cognitively healthy and between the ages of 40 and 65 years at study entry. ${ }^{20}$ Cognitive normalcy was adjudicated based on intact performance on a comprehensive battery of neuropsychological tests, absence of functional impairment, and absence of neurologic/psychiatric conditions that might impair cognition. ${ }^{20}$ All 317 participants completed a physical activity questionnaire, neuropsychological evaluations, and MRI brain scans. A subset also underwent ${ }^{11} \mathrm{C}$-Pittsburgh compound $\mathrm{B}$ $(\mathrm{PiB}, \mathrm{n}=186)$ and ${ }^{18} \mathrm{~F}$-fluorodeoxyglucose $(\mathrm{FDG}, \mathrm{n}=152)$ imaging. All participants who had PET imaging also had MRI And, with the exception of 3 individuals, all participants who had FDG imaging also had PiB imaging.

Standard protocol approvals, registrations, and patient consents. The University of Wisconsin Institutional Review Board approved all study procedures and each subject provided signed informed consent before participation.

Physical activity measurement. Participants completed the Women's Health Initiative physical activities questionnaire. ${ }^{21}$ The questionnaire assesses current frequency and duration of walking outside the home for $>10$ minutes without stopping, and of engagement in mild (e.g., slow dancing, golf), moderate (e.g., calisthenics, easy swimming), and vigorous (e.g., jogging, aerobics) exercise. For walking, frequency ranged from rarely to $\geq 7$ times/week and duration ranged from $<20$ minutes to $\geq 1$ hour/session. For mild, moderate, and vigorous exercises, frequency ranged from none to $\geq 5$ days/week whereas duration ranged from $<30$ minutes to $\geq 1$ hour/session.

Following established protocol, ${ }^{21}$ the midpoint of reported frequency and duration for each exercise category were multiplied to create respective "hours/week" measures. Next, these hours/ week measures were assigned metabolic equivalent (MET) weights as follows: for walking, casual speed $=0$, average $=3$, fast $=4$, and very fast $=4.5$; mild exercise $=3$, moderate $=4$, and vigorous $=7$. This yielded MET-hours/week measurements for each exercise intensity level. ${ }^{21}$ Finally, all 4 MET-hours/week variables were summed to create a total physical activity score. Because this total physical activity score was severely skewed even after transformation, and to make our analyses more clinically meaningful, participants were classified as physically active $(\mathrm{n}=238)$ or inactive $(\mathrm{n}=79)$ based on whether their total physical activity score was $\geq 7.5$ MET-hours/week, which approximates current American Heart Association recommendation of 30 minutes of moderate exercise for 5 days/week. ${ }^{8,9,22}$

Neuroimaging protocol. Details on the acquisition and postprocessing of the PET and MRI examinations have been previously described. ${ }^{23}$ Briefly, 3-dimensionsal PET data were acquired on a Siemens EXACT HR+ scanner (Siemens AG, Erlangen, Germany). PiB imaging consisted of a 6-minute transmission scan and a 70-minute dynamic scan upon bolus injection. FDG imaging was done per Alzheimer's Disease Neuroimaging Initiative protocol ${ }^{24}$ and involved a 30-minute scan acquired 30 minutes after bolus injection. Postprocessing for both PiB and FDG was based on an in-house automated pipeline. ${ }^{25}$ We derived distribution volume ratio (DVR) maps from the PiB images using the Logan method, with a cerebellar gray matter reference. ${ }^{26}$ Three-dimensional MRIs were acquired on a GE x750 3.0T scanner (GE Healthcare, Waukesha, WI) using a spoiled gradient recalled echo sequence. Participants completed the imaging examinations within $5.79 \pm 4.95$ months of the physical activity questionnaire.

To enhance clinical applicability and allow for the possibility of regional heterogeneity in $A \beta$ deposition within this age range when $A \beta$ burden may only be emerging, we determined amyloidosis via visual rating of the DVR maps. Specifically, after an initial assessment of a subset of images by 3 blinded raters yielded high inter- and intrarater reliability (intraclass correlations $>0.95$ ), a similarly blinded single rater visually rated all images on the intensity and pattern of cortical amyloid binding. ${ }^{23}$ Scans were deemed (1) $A \beta$ negative $(A \beta-)$ if there was no cortical $A \beta$ or only nonsignificant patchy/diffuse cortical $A \beta$ not resembling an $A D$ pattern; (2) $A \beta$ indeterminate $(A \beta i)$ if there was $P i B$ binding in at least 3 cortical lobes resembling an $A D$ pattern but less intense than an overtly positive scan; or (3) $A \beta$ positive $(A \beta+)$ when there was unambiguous, $\mathrm{AD}$-like, $\mathrm{PiB}$ binding in the cortex. ${ }^{23}$ Of the 186 individuals with $\mathrm{PiB}$ data, 74 were classified as $A \beta-, 77$ were $A \beta i$, and 35 were $A \beta+$. For FDG, we focused on the posterior cingulate cortex (PCC), a well-established inception site for AD-related neurometabolic alterations, ${ }^{19}$ and sampled FDG uptake values from it (normalized to whole-brain uptake) using the PCC mask within the Alzheimer's Disease Neuroimaging Initiative FDG Meta-ROI suite. ${ }^{27}$ We measured hippocampal volume from the MRIs using FreeSurfer version 5.1.

Neuropsychological assessment. Participants completed a comprehensive neuropsychological battery that has been previously shown to segregate into 6 cognitive factors ${ }^{28,29}$ : Immediate Memory (Rey Auditory Verbal Learning Test trials 1 and 2); Verbal Learning and Memory (Rey Auditory Verbal Learning Test trials 3-5 and Delayed Recall); Working Memory (Digit Span and LetterNumber Sequencing subtests of the Wechsler Adult Intelligence Scale-3rd edition); Speed and Flexibility (Stroop Color-Word Interference and Trail Making Test); Visuospatial Ability (Block Design and Matrix Reasoning subtests of the Wechsler Abbreviated Scale of Intelligence and Benton Judgment of Line Orientation); and Verbal Ability (Vocabulary and Similarities subtests from the Wechsler Abbreviated Scale of Intelligence, Boston Naming 
Table 1 Characteristics of study participants

\begin{tabular}{|c|c|c|c|}
\hline Variable & $\begin{array}{l}\text { Physically inactive } \\
\text { ( } n=79)\end{array}$ & $\begin{array}{l}\text { Physically active } \\
\text { ( } n=238)\end{array}$ & p Value \\
\hline \multicolumn{4}{|l|}{ Demographics } \\
\hline Age, y & $59.39(6.89)$ & $60.59(6.07)$ & 0.169 \\
\hline Female, \% & 70.9 & 67.2 & 0.545 \\
\hline Education & $15.80(2.21)$ & $16.09(2.38)$ & 0.339 \\
\hline White, \% & 96.2 & 98.3 & 0.267 \\
\hline FH positive, \% & 78.5 & 72.3 & 0.276 \\
\hline APOE $\varepsilon 4$ positive, $\%$ & 40.5 & 39.9 & 0.926 \\
\hline $\begin{array}{l}\text { Mini-Mental State } \\
\text { Examination }\end{array}$ & $29.39(0.90)$ & $29.48(0.92)$ & 0.498 \\
\hline CES-D & 7.66 (7.90) & $5.54(5.27)$ & 0.022 \\
\hline $\begin{array}{l}\text { Brain scan-physical activity } \\
\text { assessment interval, mo }\end{array}$ & $5.15(4.52)$ & $6.01(5.07)$ & 0.185 \\
\hline \multicolumn{4}{|l|}{ Vascular indices } \\
\hline Hypertension, \% & 11.4 & 13.0 & 0.670 \\
\hline Diabetes, \% & 0.0 & 1.7 & 0.575 \\
\hline Smoker, \% & 47.2 & 46.1 & 0.907 \\
\hline Taking a $\beta$-blocker, \% & 8.9 & 8.4 & 0.900 \\
\hline Taking a statin, \% & 21.5 & 24.4 & 0.605 \\
\hline Body mass index, $\mathrm{kg} / \mathrm{m}^{2}$ & 30.65 (6.29) & $27.06(4.47)$ & $<0.001$ \\
\hline $\begin{array}{l}\text { Systolic blood } \\
\text { pressure, mm Hg }\end{array}$ & 126.33 (13.78) & $123.74(16.20)$ & 0.169 \\
\hline $\begin{array}{l}\text { Diastolic blood } \\
\text { pressure, } \mathrm{mm} \mathrm{Hg}\end{array}$ & 75.04 (8.93) & $73.40(9.61)$ & 0.183 \\
\hline Total cholesterol, mg/dL & 196.68 (34.77) & 196.07 (33.91) & 0.889 \\
\hline HDL cholesterol, mg/dL & $54.65(13.65)$ & $61.65(17.70)$ & $<0.001$ \\
\hline Homocysteine, $\mu \mathrm{mol} / \mathrm{L}$ & $9.32(3.86)$ & $9.16(2.48)$ & 0.671 \\
\hline Creatinine, mg/dL & $0.86(0.16)$ & $0.88(0.18)$ & 0.231 \\
\hline hs $\mathrm{C}$-reactive protein, $\mathrm{mg} / \mathrm{L}$ & 2.98 (3.28) & $2.31(4.35)$ & 0.211 \\
\hline HOMA-IR & $2.76(2.41)$ & $2.03(1.76)$ & 0.014 \\
\hline Interleukin-6, pg/mL & $2.10(1.65)$ & 1.99 (1.95) & 0.679 \\
\hline \multicolumn{4}{|l|}{ Imaging biomarkers } \\
\hline \multicolumn{4}{|l|}{ PiB-PET visual rating, ${ }^{a} \%$} \\
\hline$A \beta-$ & 51.9 & 35.1 & 0.094 \\
\hline $\mathbf{A} \beta \mathbf{i}$ & 30.8 & 45.5 & \\
\hline$A \beta+$ & 17.3 & 19.4 & \\
\hline PiB-PET DVR ${ }^{a}$ & $1.12(0.11)$ & $1.13(0.13)$ & 0.510 \\
\hline FDG-PET, ${ }^{a} \mathrm{nCi} / \mathrm{mL}$ & $82,716.66(16,004.42)$ & $86,587.46(20,367.67)$ & 0.274 \\
\hline Hippocampal volume, $\mathrm{mm}^{3}$ & $3,942.94$ (411.16) & $3,935.30(424.25)$ & 0.889 \\
\hline
\end{tabular}

Abbreviations: $A \beta=\beta$-amyloid; $A \beta i=A \beta$ indeterminate; $C E S-D=$ Center for Epidemiologic Studies Depression Scale; DVR = distribution volume ratio; FDG $={ }^{18} \mathrm{~F}$-fluorodeoxyglucose; $\mathrm{FH}=$ parental family history of Alzheimer disease; HDL = high-density lipoprotein; HOMA-IR $=$ Homeostasis Model Assessment of Insulin Resistance (i.e., [glucose $\times$ insulin]/ 405]); hs = high-sensitivity; PiB $={ }^{11} \mathrm{C}$-Pittsburgh compound B.

Values are mean (SD) unless otherwise specified.

${ }^{a}$ For $P i B$, total $n=186$, physically inactive $n=52$, physically active $n=134$; for FDG, total $n=152$, physically inactive $n=41$, physically active $n=111$. PiB-PET DVR data were extracted from a precuneus region of interest. FDG-PET data were extracted from a posterior cingulate region of interest.
Test, and Reading subtest of the Wide-Range Achievement Test3rd edition). To minimize multiple comparisons, these factor scores, rather than the component cognitive tests, were interrogated in the present analyses. Participants completed these cognitive tests during the same study visit as the physical activity questionnaire.

Vascular risk factors. Participants completed a health history questionnaire that included questions about cardiovascular disease. They also underwent a clinic visit that comprised anthropometric measurements, blood pressure readings, and phlebotomy for laboratory tests implicated in vascular disease.

Statistical analyses. Group differences on demographic and vascular measures were tested using independent samples $t$ test or $\chi^{2}$ analyses. To determine the relative influence of age, $\mathrm{FH}$, and $A P O E$ $\varepsilon 4$ status on our imaging and cognitive measures, we fitted a series of linear (for hippocampal volume, FDG, and cognitive factors) or logistic (for PiB rating) regression models that were adjusted for relevant covariates such as sex, education, intracranial volume, and global FDG uptake as applicable. To address the primary aim of this study, i.e., the modulation of age-associated alterations in $\mathrm{AD}$ biomarkers by physical activity, we fitted covariate-adjusted linear or logistic regression models in which the term of primary interest was the interaction between age and level of physical activity (i.e., active vs inactive). Where significant, this term would indicate that the deleterious effect of age on these biomarkers is modified by physical activity. To facilitate the interpretability of the interaction terms, age was treated as a dichotomy ( $<60$ [younger] vs $\geq 60$ [older]) during model fitting. In addition, for all the logistic regression models, we collapsed the $A \beta-$ and $A \beta i$ groups into one group such that the regression coefficients indicated the likelihood of being unambiguously $A \beta+$. Alternative groupings (e.g., $A \beta+$ vs $A \beta-, A \beta+$ vs $A \beta i$ vs $A \beta-$ ) were explored and results were not substantively changed. All analyses were performed using SPSS 20.0 (IBM Corp., Armonk, NY) or SAS 9.2 (SAS Institute, Cary, NC). Only findings with a 2-tailed $p$ value $\leq 0.05$ were considered significant.

RESULTS Background characteristics. The groups did not differ on any demographic variable or imaging biomarker. Although the inactive group had higher depressive symptoms, their mean score on this measure was well below the cutpoint (i.e., 16) for clinical depression. ${ }^{30}$ The inactive group also exhibited worse scores on measures of body habitus, high-density lipoprotein, and insulin resistance (table 1). Our primary analyses were rerun, controlling for these 4 potentially confounding factors.

$\mathrm{AD}$ risk factors and interindividual variation in $\mathrm{AD}$ biomarkers. As expected, age made a greater contribution to between-person variability in all biomarkers compared with $\mathrm{FH}$ and $A P O E$ \&4. The only exceptions were the Verbal Ability composite (wherein FH was more influential) and the Working Memory composite, which was not influenced by any of the 3 risk factors (table 2). To further validate these findings, especially given the apparently modest influence of $\mathrm{FH}$ and $A P O E \& 4$, the data were reanalyzed using a series of 2-block hierarchical regression in which relevant covariates (e.g., sex and 
Table 2 Influence of age, $\mathrm{FH}$, and $\mathrm{APOE} \varepsilon 4$ on interindividual variability in $\mathrm{AD}$ biomarkers

\begin{tabular}{|c|c|c|c|c|c|c|}
\hline Biomarker & \multicolumn{2}{|l|}{ Age } & \multicolumn{2}{|l|}{$\mathrm{FH}$} & \multicolumn{2}{|l|}{ APOE $\& 4$} \\
\hline $\mathrm{PiB}^{\mathrm{a}}$ & $0.14(0.04)$ & 0.001 & $0.78(0.52)$ & 0.130 & $1.06(0.43)$ & 0.013 \\
\hline Hippocampal volume ${ }^{c}$ & -21.32 (3.39) & $<0.001$ & 6.66 (48.98) & 0.892 & $-54.93(43.64)$ & 0.209 \\
\hline Immediate Memory ${ }^{d}$ & $-0.03(0.01)$ & 0.001 & $-0.15(0.13)$ & 0.261 & $-0.05(0.12)$ & 0.656 \\
\hline Speed and Flexibility ${ }^{d}$ & $-0.07(0.01)$ & $<0.001$ & $-0.01(0.11)$ & 0.924 & $-0.06(0.10)$ & 0.563 \\
\hline Working Memory ${ }^{d}$ & $-0.02(0.01)$ & 0.126 & $-0.07(0.14)$ & 0.639 & $-0.20(0.12)$ & 0.111 \\
\hline Visuospatial Ability $^{d}$ & $-0.05(0.01)$ & $<0.001$ & $-0.05(0.11)$ & 0.649 & $-0.03(0.10)$ & 0.732 \\
\hline Verbal Ability ${ }^{d}$ & $0.00(0.01)$ & 0.743 & $-0.31(0.11)$ & 0.004 & $0.10(0.09)$ & 0.271 \\
\hline
\end{tabular}

Abbreviations: $A D=$ Alzheimer disease; $\beta=$ regression estimate; $F D G={ }^{18} F$-fluorodeoxyglucose; $F H=$ parental family history of $A D ; P i B={ }^{11} C-P i t t s b u r g h$ Compound B; SE = standard error.

Sample sizes for the analyses were 317 for hippocampal volume and cognition, 186 for PiB, and 152 for FDG.

a Variables included in the statistical model were sex, age, $\mathrm{FH}$, and APOE $\varepsilon 4$.

${ }^{b}$ Variables included in the statistical model were sex, global FDG, age, $\mathrm{FH}$, and APOE $\varepsilon 4$.

${ }^{\mathrm{c}}$ Variables included in the statistical model were sex, intracranial volume, age, $\mathrm{FH}$, and $A P O E \& 4$.

${ }^{d}$ Variables included in the statistical model were sex, education, age, $\mathrm{FH}$, and APOE $\varepsilon 4$.

global FDG, for the PCC FDG model) were forceentered in block 1, with age, $\mathrm{FH}$, and $A P O E \varepsilon 4$ stepwise-entered in block 2. Results from this set of analyses were consistent with the original findings, i.e., across all models, age was the only variable selected for entry in block 2, with the following exceptions: for PiB, APOE $\varepsilon 4$ was also selected for entry after age; for Verbal Ability, only FH was selected for entry; and for Working Memory, neither age, nor $\mathrm{FH}$, nor $A P O E \& 4$ was selected.

Physical activity ameliorates age-associated biomarker alterations. Table 3 and the figure present the results of the analyses that investigated whether physical activity attenuates the deleterious effect of age on $\mathrm{AD}$ biomarkers. The linear regression models revealed age $X$ physical activity interaction effects for FDG, hippocampal volume, Immediate Memory, Visuospatial Ability, and a trend for Verbal Ability. Follow-up simple main effects analyses revealed 2 patterns of within-group age effects, all favoring the active group. Pattern A (observed for FDG and Immediate Memory) consisted of an age effect, e.g., increased age associated with reduced FDG, within the inactive group, that was not seen within the active group. Pattern B (observed for hippocampal volume and Visuospatial Ability) consisted of an age effect, e.g., increased age associated with decreased hippocampal volume, within both groups. However, this effect was attenuated in the active group $(43 \%$ less for hippocampal volume and 38\% less for Visuospatial Ability) relative to the inactive group. Of note, the significance of the age $\times$ physical activity interaction term in each model remained essentially unaltered after additional adjustment was made for the potentially confounding vascular and mood measures: FDG $(p=0.015)$, hippocampal volume $(p=0.021)$, Immediate Memory ( $p=0.051$; originally 0.042$)$, and Visuospatial Ability $(p=0.019)$.

The logistic regression model for determining whether physical activity attenuates the association between age and unambiguous $A \beta$ aggregation was fit with the Firth penalized likelihood method ${ }^{31}$ because of the quasi-separation in the dataset caused by a cell (younger, inactive, and $A \beta+$ ) with 0 count. As recommended, ${ }^{31}$ statistical significance was assessed using likelihood ratio testing. The age $\times$ physical activity term was statistically significant $\left(\Delta \chi^{2}{ }_{1}=6.03, p=\right.$ $0.014)$. Follow-up simple main effects analyses revealed that, within the inactive group, increased age was associated with higher odds of being $\mathrm{A} \beta+$, whereas within the active group, the age effect was absent (i.e., pattern A finding). This finding persisted upon adjustment for the 4 potentially confounding covariates noted earlier. We repeated the PiB analyses with quantitative DVR data extracted using a 3-mm-radius sphere placed around the nexus of most overt amyloidosis in our cohort (MNI x, y, z coordinates $[-8,-66,52]$, precuneus). ${ }^{32}$ We observed an age $\times$ physical activity effect (B $[S E]=-0.63[0.32], p=0.047)$, with simple main effects analyses showing that increased age was associated with greater $\mathrm{PiB}$ binding in the inactive group $(\mathrm{B}[\mathrm{SE}]=0.92[0.26], p=0.001)$ but not in the active group $(\mathrm{B}[\mathrm{SE}]=0.29[0.15], p=0.100)$.

Lastly, as exploratory analyses, we deleted the age $\times$ physical activity term from those models 
Table 3 Physical activity attenuates age-related alterations in AD biomarkers

\begin{tabular}{|c|c|c|c|c|c|c|}
\hline Biomarker & \multicolumn{2}{|c|}{ Age $\times$ physical activity $^{a}$} & \multicolumn{2}{|l|}{ Age $_{(\text {inactive) }}{ }^{b}$} & \multicolumn{2}{|l|}{ Age $_{(\text {active) }}{ }^{c}$} \\
\hline $\mathrm{PiB}^{\mathrm{d}, \mathrm{e}}$ & $-2.95(1.59)$ & 0.014 & $3.61(1.47)$ & 0.013 & $0.74(0.50)$ & 0.140 \\
\hline FDGe,f & 4,945.83 (1,999.26) & 0.015 & $-4,379.60(1,763.52)$ & 0.018 & $1,133.06(1,048.56)$ & 0.282 \\
\hline Hippocampal volume $e^{e, g}$ & 222.69 (99.02) & 0.025 & $-385.44(71.82)$ & $<0.001$ & $-165.93(52.31)$ & 0.002 \\
\hline Immediate Memory ${ }^{\mathrm{e}, \mathrm{h}}$ & $0.53(0.26)$ & 0.042 & $-0.63(0.22)$ & 0.006 & $-0.16(0.13)$ & 0.232 \\
\hline Speed and Flexibility ${ }^{e, h}$ & $0.19(0.22)$ & 0.389 & - & - & - & - \\
\hline Working Memory $y^{e, h}$ & $0.35(0.27)$ & 0.196 & - & - & - & - \\
\hline Visuospatial Ability e,h & $0.53(0.22)$ & 0.016 & $-0.88(0.18)$ & $<0.001$ & $-0.33(0.11)$ & 0.003 \\
\hline Verbal Ability ${ }^{\text {e,h }}$ & $0.35(0.21)$ & 0.094 & - & - & - & - \\
\hline
\end{tabular}

Abbreviations: $A D=$ Alzheimer disease; $\beta=$ regression estimate; FDG $={ }^{18} \mathrm{~F}$-fluorodeoxyglucose; $\mathrm{PiB}={ }^{11} \mathrm{C}-\mathrm{Pittsburgh}$ Compound $\mathrm{B} ; \mathrm{SE}=\mathrm{standard}$ error . Sample sizes for the analyses were 317 for hippocampal volume and cognition, 186 for PiB, and 152 for FDG.

${ }^{a}$ The regression estimates and associated $p$ values are for the age $\times$ physical activity interactive term in each biomarker's model. This term assesses whether physical activity modifies the effect of age on the examined biomarker.

${ }^{b}$ The regression estimates and associated $p$ values are for the simple main effect of age on each biomarker within the inactive group.

${ }^{\mathrm{c}}$ The regression estimates and associated $p$ values are for the simple main effect of age on each biomarker within the active group.

${ }^{d}$ Variables included in the full model were sex, age, physical activity, and age $\times$ physical activity. Variables included in the simple main effects model were sex and age.

e Statistical significance for the age $\times$ physical activity term in each full model remained essentially unchanged after additional adjustment was made for depressive symptoms, body mass index, high-density lipoprotein, and insulin resistance.

${ }^{f}$ Variables included in the full model were sex, global FDG, age, physical activity, and age $\times$ physical activity. Variables included in the simple main effects model were sex, global FDG, and age.

${ }^{g}$ Variables included in the full model were sex, intracranial volume, age, physical activity, and age $\times$ physical activity. Variables included in the simple main effects model were sex, intracranial volume, and age.

${ }^{h}$ Variables included in the full model were sex, education, age, physical activity, and age $\times$ physical activity. Variables included in the simple main effects model were sex, education, and age.

wherein it was null (i.e., Verbal Learning and Memory, Speed and Flexibility, Working Memory, and Verbal Ability) and refit the model to assess the main effect of physical activity. We observed an effect for only Speed and Flexibility $(\beta[S E]=0.35[0.11]$, $p=0.002)$, wherein the active group had higher scores compared with the inactive group.

DISCUSSION This study showed that, in a latemiddle-aged cohort at risk of $\mathrm{AD}$, physically active individuals experienced less age-related alterations in $\mathrm{A} \beta$ deposition, glucose metabolism, hippocampal volume, Immediate Memory, and Visuospatial Ability compared with physically inactive persons. These internally replicated findings are novel given that no prior studies have tested whether physical activity might attenuate the adverse relationship between age and putative $\mathrm{AD}$ pathology using an array of core $\mathrm{AD}$ biomarkers and a unique cohort of at-risk middle-aged adults. Even so, we note that we are unable to definitively establish the directionality of these observed effects given the observational, cross-sectional nature of our design. This study also found that age was, overall, more influential than $\mathrm{FH}$ and $A P O E \& 4$ in explaining interindividual variation in these $\mathrm{AD}$ biomarkers.
A large body of work, including observational and intervention studies, has shown that physical activity is beneficial for maintaining cognitive function and delaying the onset of $\mathrm{AD}$ and related diseases among older adults. ${ }^{4,33}$ However, it is only recently that studies have begun to investigate the potential effects of physical activity on biological markers of the pathophysiologic cascade associated with $\mathrm{AD}$. A landmark study $^{6}$ demonstrated that aerobic training engenders increased hippocampal volume, confirming initial reports of augmented hippocampal angiogenesis/neurogenesis $^{34}$ and perfusion ${ }^{35}$ after structured aerobic training. Other investigations $s^{5,7}$ have similarly reported associations between cardiorespiratory fitness, an index of habitual physical activity, and hippocampal volume.

Although hippocampal atrophy is a common phenotype of $\mathrm{AD}$, it is not pathognomonic given that it is also observed in other clinical syndromes. ${ }^{36,37}$ Therefore, more recently, there has been an interest in determining whether physical activity modulates more elemental hallmarks of $A D$, such as $A \beta$ aggregation. Some studies found that older adults who frequently engaged in physical activity exhibited reduced $A \beta^{8-10}$ and tau $^{8}$ abnormalities compared with their less active peers, whereas other reports ${ }^{11,12}$ 

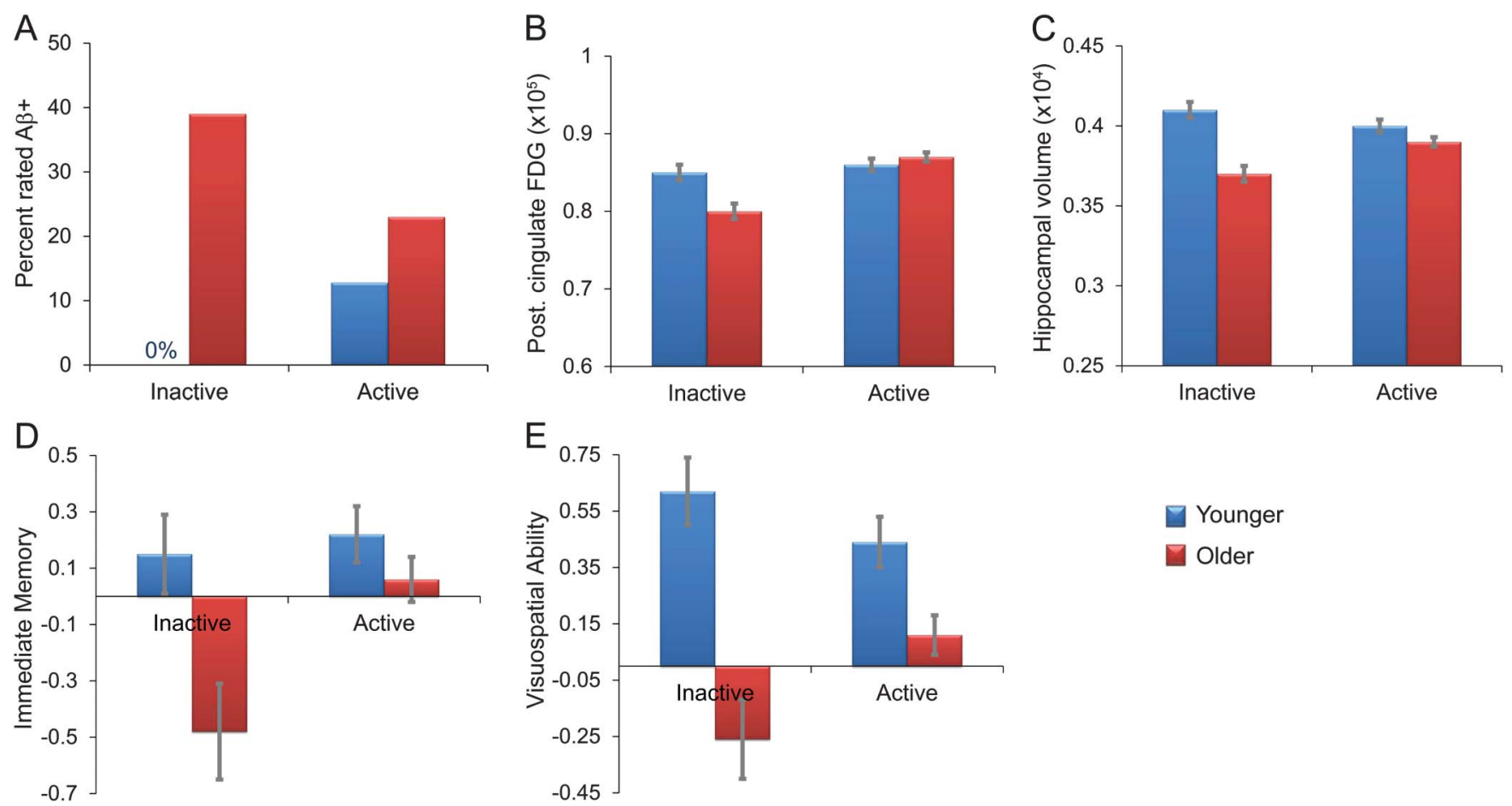

Younger

Older

Blue bars = younger participants (i.e., $<60$ years); red bars = older participants (i.e., $\geq 60$ years). (A) Percentage of participants whose PiB scans were rated as being unambiguously positive for $A \beta$ in an AD-like pattern. (B-E) adjusted means and standard error for (B) FDG posterior cingulate glucose metabolism, (C) hippocampal volume, (D) Immediate Memory, and (E) Visuospatial Ability. Sample sizes for the analyses were 317 for hippocampal volume and cognition, 186 for PiB, and 152 for FDG. Cell counts are as follows: for hippocampal volume and cognition, inactive and younger $n=45$, inactive and older $n=34$, active and younger $n=95$, and active and older $n=143$; for $P i B$, inactive and younger $n=29$, inactive and older $n=23$, active and younger $n=47$, and active and older $n=87$; and for FDG, inactive and younger $n=23$, inactive and older $n=18$, active and younger $n=43$, and active and older $n=68$. $A \beta=$ $\beta$-amyloid; $A D=$ Alzheimer disease; FDG $={ }^{18} \mathrm{~F}$-fluorodeoxyglucose; $\mathrm{PiB}={ }^{11} \mathrm{C}$-Pittsburgh compound $\mathrm{B}$.

failed to detect such effects. Our study extends these earlier investigations in several important ways. First, we studied a unique, well-characterized cohort of latemiddle-aged adults, the majority of whom harbor key risk factors for $\mathrm{AD}(73.8 \%$ with $\mathrm{FH}$ of $\mathrm{AD}$ and $40.1 \%$ APOE $\varepsilon 4$ positive). Second, compared with these prior studies, which mostly focused on $A \beta$, our study examined an array of the major biomarkers of AD pathophysiology. ${ }^{19}$ Third, our sample size is considerably larger than most prior studies, conferring added validity to our findings. Finally, this study shows novel associations indicative of an ameliorative role for physical activity vis-à-vis age-related pathophysiologic alterations in the $\mathrm{AD}$ cascade. These associations provide confirmatory support for earlier preliminary reports suggesting that physical activity retards aging-induced brain atrophy in older adults. ${ }^{13,14}$

Not surprisingly, our examination of the comparative influence of age, $\mathrm{FH}$, and $A P O E \& 4$ on the $\mathrm{AD}$ biomarkers revealed that age explained a greater proportion of the interindividual variation in these biomarkers. Although, to our knowledge, no prior study has directly investigated the relative degree to which these 3 cardinal risk factors for $\mathrm{AD}$ influence in vivo biomarker abnormalities, prior epidemiologic and autopsy studies have highlighted the singular role that age plays in $\mathrm{AD}$ incidence and neuropathology. For example, an early population-based study revealed that the age-specific incidence of $\mathrm{AD}$ is nearly 14 times higher in the 85 years and older age group compared with the 65 to 69 group, ${ }^{17}$ a finding that has since been replicated in several studies. ${ }^{18}$ Similarly, autopsy studies have demonstrated that there is a strong age gradient to the frequency and severity of $A \beta$ deposits and neurofibrillary tangles, ${ }^{15}$ and that the effect of $A P O E \& 4$ on these histologic markers is likely confounded with age. ${ }^{38} \mathrm{~A}$ full elucidation of the mechanism of action underlying this robust relationship between age and the clinicopathologic features of AD lies beyond the scope of this study. In one theoretical framework, ${ }^{39} \mathrm{AD}$ is seen as resulting from the intricate interactions among multiple age-related molecular changes, some programmed and others stochastic, that ultimately engender increased entropy. This elevated entropy is considered the final common pathway to the progressive neural and cognitive decline that characterizes AD. ${ }^{16,39}$ Such theoretical models might 
prove useful for generating testable hypotheses that further our understanding of the strong covariation between age and the clinical and pathologic features of AD.

A key limitation of this study is its cross-sectional design. Although we have used statistical approaches to estimate the potential for physical activity to ameliorate age-associated pathophysiologic changes within the $\mathrm{AD}$ cascade, a truly prospective design (especially in the context of a randomized controlled trial), will be critical to evaluating whether physical activity exerts a causal influence on cognitive/biomarker trajectories in people at risk of AD. Such longitudinal data, as are being collected in the WRAP cohort, will also provide needed evidence concerning rates of progression to mild cognitive impairment/ dementia among asymptomatic middle-aged adults who engage in differential levels of physical activity. Relatedly, our cross-sectional design does not permit us to exclude the possibility of reverse causality (i.e., that favorable biomarker profiles enable physical activity). We also acknowledge that the demographic composition of our cohort might limit the generalizability of our study findings. Lastly, self-reported measures are vulnerable to various sources of bias. Objective measures of physical activity and fitness, such as accelerometry and graded exercise testing, would likely provide more valid estimates of the influence of physical activity on cognitive/biomarker trajectories.

Overall, this study demonstrates that the deleterious effect of aging on $\mathrm{AD}$ biomarkers and cognition is attenuated among physically active middle-aged adults at risk for $\mathrm{AD}$ compared with their less active peers. If these findings are supported by prospective, controlled studies, it would provide compelling evidence for physical activity as an efficacious approach to $\mathrm{AD}$ prevention, particularly in risk-enriched cohorts.

\section{AUTHOR CONTRIBUTIONS}

Drafting/revising the manuscript for content: Okonkwo, Oh, Schultz, Larson, Edwards, Cook, Koscik, Gallagher, Dowling, Carlsson, Bendlin, LaRue, Rowley, Christian, Asthana, Hermann, Johnson, Sager. Study concept or design: Okonkwo, Christian, Asthana, Hermann, Johnson, Sager. Analysis or interpretation of data: Okonkwo, Oh, Schultz, Larson, Edwards, Cook, Koscik, Gallagher, Dowling, Carlsson, Bendlin, LaRue, Rowley, Christian, Asthana, Hermann, Johnson, Sager. Acquisition of data: Oh, Edwards, Koscik, LaRue, Rowley, Christian, Asthana, Hermann, Johnson, Sager. Statistical analyses: Okonkwo. Study supervision or coordination: Okonkwo, Hermann, Johnson, and Sager. Obtaining funding: Okonkwo, Asthana, Johnson, Sager.

\section{ACKNOWLEDGMENT}

Special thanks to Todd E. Barnhart, PhD, Dhanabalan Murali, PhD, Dustin Wooten, PhD, Ansel Hillmer, MSc, Lance T. Hall, MD, Guofan Xu, $\mathrm{MD}, \mathrm{PhD}$, and Andrew Higgins for assistance with PET data production, processing, and visual rating; Caitlin A. Cleary, BSc, Sandra Harding, MS, Jennifer Bond, BA, Janet Rowley, BA, and the WRAP psychometrists for helping with study data collection; researchers and staff at the Waisman
Center, University of Wisconsin-Madison, where the brain scans took place; and especially participants in the Wisconsin Registry for Alzheimer's Prevention for their continued dedication.

\section{STUDY FUNDING}

This work was supported by National Institute on Aging grants K23 AG045957 (O.C.O.), R01 AG027161 (M.A.S.), R01 AG021155 (S.C.J.), P50 AG033514 (S.A.), and P50 AG033514-S1 (O.C.O.); by a Veterans Administration Merit Review Grant I01CX000165 (S.C.J.); and by a Clinical and Translational Science Award (UL1RR025011) to the University of Wisconsin, Madison. Portions of this research were supported by the Wisconsin Alumni Research Foundation, the Helen Bader Foundation, Northwestern Mutual Foundation, Extendicare Foundation, and from the Veterans Administration including facilities and resources at the Geriatric Research Education and Clinical Center of the William S. Middleton Memorial Veterans Hospital, Madison, WI. The funders' role was limited to providing funding for the study.

\section{DISCLOSURE}

The authors report no disclosures relevant to the manuscript. Go to Neurology.org for full disclosures.

Received April 15, 2014. Accepted in final form August 6, 2014.

\section{REFERENCES}

1. Adlard PA, Perreau VM, Pop V, Cotman CW. Voluntary exercise decreases amyloid load in a transgenic model of Alzheimer's disease. J Neurosci 2005;25: 4217-4221.

2. Fuss J, Biedermann SV, Falfan-Melgoza C, et al. Exercise boosts hippocampal volume by preventing early agerelated gray matter loss. Hippocampus 2014;24: 131-134.

3. Marlatt MW, Potter MC, Lucassen PJ, van Praag H. Running throughout middle-age improves memory function, hippocampal neurogenesis, and BDNF levels in female C57BL/6J mice. Dev Neurobiol 2012;72:943-952.

4. Angevaren M, Aufdemkampe G, Verhaar HJ, Aleman A, Vanhees L. Physical activity and enhanced fitness to improve cognitive function in older people without known cognitive impairment. Cochrane Database Syst Rev 2008; (3):CD005381

5. Honea RA, Thomas GP, Harsha A, et al. Cardiorespiratory fitness and preserved medial temporal lobe volume in Alzheimer disease. Alzheimer Dis Assoc Disord 2009;23: 188-197.

6. Erickson KI, Voss MW, Prakash RS, et al. Exercise training increases size of hippocampus and improves memory. Proc Natl Acad Sci USA 2011;108: 3017-3022.

7. Erickson KI, Prakash RS, Voss MW, et al. Aerobic fitness is associated with hippocampal volume in elderly humans. Hippocampus 2009;19:1030-1039.

8. Liang KY, Mintun MA, Fagan AM, et al. Exercise and Alzheimer's disease biomarkers in cognitively normal older adults. Ann Neurol 2010;68:311-318.

9. Head D, Bugg JM, Goate AM, et al. Exercise engagement as a moderator of the effects of APOE genotype on amyloid deposition. Arch Neurol 2012;69:636-643.

10. Brown BM, Peiffer JJ, Taddei K, et al. Physical activity and amyloid-beta plasma and brain levels: results from the Australian Imaging, Biomarkers and Lifestyle Study of Ageing. Mol Psychiatry 2013;18:875-881.

11. Landau SM, Marks SM, Mormino EC, et al. Association of lifetime cognitive engagement and low beta-amyloid deposition. Arch Neurol 2012;69:623-629. 
12. Vemuri P, Lesnick TG, Przybelski SA, et al. Effect of lifestyle activities on Alzheimer disease biomarkers and cognition. Ann Neurol 2012;72:730-738.

13. Bugg JM, Head D. Exercise moderates age-related atrophy of the medial temporal lobe. Neurobiol Aging 2011;32: 506-514.

14. Colcombe SJ, Erickson KI, Raz N, et al. Aerobic fitness reduces brain tissue loss in aging humans. J Gerontol A Biol Sci Med Sci 2003;58:176-180.

15. Braak H, Braak E. Frequency of stages of Alzheimerrelated lesions in different age categories. Neurobiol Aging 1997; 18:351-357.

16. Savva GM, Wharton SB, Ince PG, Forster G, Matthews FE, Brayne C. Age, neuropathology, and dementia. N Engl J Med 2009;360:2302-2309.

17. Hebert LE, Scherr PA, Beckett LA, et al. Age-specific incidence of Alzheimer's disease in a community population. JAMA 1995;273:1354-1359.

18. Mayeux R, Stern Y. Epidemiology of Alzheimer disease. Cold Spring Harb Perspect Med 2012;2:a006239.

19. Sperling RA, Aisen PS, Beckett LA, et al. Toward defining the preclinical stages of Alzheimer's disease: recommendations from the National Institute on Aging-Alzheimer's Association workgroups on diagnostic guidelines for Alzheimer's disease. Alzheimers Dement 2011;7:280-292.

20. Sager MA, Hermann B, La Rue A. Middle-aged children of persons with Alzheimer's disease: APOE genotypes and cognitive function in the Wisconsin Registry for Alzheimer's Prevention. J Geriatr Psychiatry Neurol 2005;18:245-249.

21. McTiernan A, Kooperberg C, White E, et al. Recreational physical activity and the risk of breast cancer in postmenopausal women: the Women's Health Initiative Cohort Study. JAMA 2003;290:1331-1336.

22. Nelson ME, Rejeski WJ, Blair SN, et al. Physical activity and public health in older adults: recommendation from the American College of Sports Medicine and the American Heart Association. Med Sci Sports Exerc 2007; 39:1435-1445.

23. Johnson SC, Christian BT, Okonkwo OC, et al. Amyloid burden and neural function in people at risk for Alzheimer's disease. Neurobiol Aging 2014;35:576-584.

24. Jagust WJ, Bandy D, Chen K, et al. The Alzheimer's Disease Neuroimaging Initiative positron emission tomography core. Alzheimers Dement 2010;6:221-229.

25. Floberg JM, Mistretta CA, Weichert JP, Hall LT, Holden JE, Christian BT. Improved kinetic analysis of dynamic PET data with optimized HYPR-LR. Med Phys 2012;39:3319-3331.
26. Price JC, Klunk WE, Lopresti BJ, et al. Kinetic modeling of amyloid binding in humans using PET imaging and Pittsburgh compound-B. J Cereb Blood Flow Metab 2005;25:1528-1547.

27. Landau SM, Harvey D, Madison CM, et al. Associations between cognitive, functional, and FDG-PET measures of decline in AD and MCI. Neurobiol Aging 2011;32: 1207-1218.

28. Dowling NM, Hermann B, La Rue A, Sager MA. Latent structure and factorial invariance of a neuropsychological test battery for the study of preclinical Alzheimer's disease. Neuropsychology 2010;24:742-756.

29. Koscik RL, La Rue A, Jonaitis EM, et al. Emergence of mild cognitive impairment in late middle-aged adults in the Wisconsin Registry for Alzheimer's Prevention. Dement Geriatr Cogn Disord 2014;38:16-30.

30. Radloff LS. The CES-D Scale: a self-report depression scale for research in the general population. Appl Psychol Meas 1977;1:385-401.

31. Heinze G, Schemper M. A solution to the problem of separation in logistic regression. Stat Med 2002;21: 2409-2419.

32. Okonkwo OC, Oh JM, Koscik R, et al. Amyloid burden, neuronal function, and cognitive decline in middle-aged adults at risk for Alzheimer's disease. J Int Neuropsychol Soc 2014;20:422-433.

33. Barnes DE, Yaffe K. The projected effect of risk factor reduction on Alzheimer's disease prevalence. Lancet Neurol 2011;10:819-828.

34. Pereira AC, Huddleston DE, Brickman AM, et al. An in vivo correlate of exercise-induced neurogenesis in the adult dentate gyrus. Proc Natl Acad Sci USA 2007;104: 5638-5643.

35. Burdette JH, Laurienti PJ, Espeland MA, et al. Using network science to evaluate exercise-associated brain changes in older adults. Front Aging Neurosci 2010;2:23.

36. Nelson PT, Smith CD, Abner EL, et al. Hippocampal sclerosis of aging, a prevalent and high-morbidity brain disease. Acta Neuropathol 2013;126:161-177.

37. DeCarolis NA, Eisch AJ. Hippocampal neurogenesis as a target for the treatment of mental illness: a critical evaluation. Neuropharmacology 2010;58:884-893.

38. Berg L, McKeel DW Jr, Miller JP, et al. Clinicopathologic studies in cognitively healthy aging and Alzheimer's disease: relation of histologic markers to dementia severity, age, sex, and apolipoprotein E genotype. Arch Neurol 1998;55:326-335.

39. Drachman DA. Aging of the brain, entropy, and Alzheimer disease. Neurology 2006;67:1340-1352.

\section{You're Committed to Continually Expanding Your Knowledge}

NeuroSAETM is committed to helping you. The Sixth Edition of the AAN's convenient online selfassessment examination is now available, featuring 150 questions and 8 self-assessment CME credits upon successful completion to help meet ABPN MOC requirements.

See all the available NeuroSAE versions and purchase the exam today at $A A N$.com/view/neuroSAE. 